\title{
Herederos de Juan Guas. Arquitectos de San Juan de Los Reyes en los siglos XIX y XX
}

\author{
Daniel OrTIZ PRADAS \\ Departamento de Historia del Arte I (Medieval) \\ Universidad Complutense de Madrid \\ dopradas@ghis.ucm.es
}

\begin{abstract}
RESUMEN
La construcción de San Juan de los Reyes de Toledo fue un proceso largo y continuado que duró algo más de cinco siglos. Durante este tiempo se han sucedido maestros y arquitectos que han imprimido su personal huella pero siempre manteniendo la unidad estilística y la obra original de Juan Guas. Este hecho resulta especialmente visible en las actuaciones de los siglos XIX y XX, sobre las que se centra este artículo. En primer lugar sobre la obra proyectada por Arturo Mélida entre 1881 y 1902, marcada por el espíritu historicista de la época y continuada después con las diferentes intervenciones de Zabala, Carrasco y Muñoz, Moya o Valcárcel en el XX promovidas por la Dirección General de Regiones Devastadas.
\end{abstract}

Palabras clave: arquitectura, Juan Guas, restauración, Mélida, Valcárcel, Regiones Devastadas.

\begin{abstract}
The construction of the monastery of San Juan de los Reyes, in Toledo, was a long process which took more of five centuries to be finished. Throughout this period, different architects have contributed, leaving their personal mark, keeping the stylistic unity and the original Juan Guas' work. This is specially clear in the additions, made in the 19th and 20th centuries; this article focuses on. First, in the project made by Arturo Mélida, between 1881 and 1902, marked by the historicist spirit of that time, and afterwards, in the different additions made by Zabala, Carraco y Muñoz, Mora or Valcárcel in the 20thcentury, promoted by the Dirección General de Regiones Devastadas.
\end{abstract}

Keywords: architecture, Juan Guas, restoration, Mélida, Valcárcel, Regiones Devastadas.

Uno de los ejemplos más significativos de la arquitectura tardogótica castellana es, sin lugar a dudas, el convento toledano de San Juan de los Reyes, el cual, según narran las fuentes, fue fundado por Isabel la Católica en 1476 en cumplimiento de un voto en agradecimiento a Dios por la victoria lograda por su marido en la batalla de Toro contra Portugal, esencial en su pugna por el derecho a la Corona de Castilla.

Obra de indiscutible belleza, supone un referente del Toledo monumental que no puede ni debe dejarse de lado en toda visita a la ciudad. Se ha dicho en multitud de ocasiones que San Juan de los Reyes es "una joya de nuestra arquitectura gótica" y citas más actuales describen sin tapujos su claustro como "uno de los más bellos de toda Europa". Dicha afirmación resulta innegable, si bien no debemos olvidar que el edificio ha estado en constante proceso de construcción, reforma o restau- 
ración desde el siglo XV hasta nuestros días y con especial intensidad durante los siglos XIX y XX. Estas transformaciones no han sido habitualmente tenidas en cuenta en la historiografía contemporánea -y cuando lo ha hecho ha sido siempre de soslayo- y ello a pesar de su trascendental importancia en la configuración del monumento medieval. Por este motivo se hacía necesaria una revisión documental e historiográfica centrada en estos dos últimos siglos. Este trabajo tratará, en este sentido, sobre las intervenciones más relevantes llevadas a cabo en San Juan de los Reyes desde 1881, fecha en la que se da comienzo su restauración, hasta los últimos años del siglo XX. Pero para ello es preciso realizar previamente un breve repaso por las diferentes fases constructivas del edificio, especialmente las menos conocidas, es decir, las de época moderna, para establecer los límites entre la obra considerada tradicionalmente histórica y la nueva. En cualquier caso es interesante señalar cómo, a pesar de los siglos transcurridos y la sucesión de arquitectos que participaron en la construcción de San Juan de los Reyes, ha existido siempre un decidido propósito de mantener la unidad de estilo del edificio y con ella la obra de Guas.

\section{Primeras etapas constructivas: de la fundación al siglo XIX}

La historia de la edilicia del monumento está bastante bien documentada, por tanto no entraremos en detalles relativos a sus primeros años de construcción; desde las primeras trazas de la iglesia y claustro de Juan Guas en colaboración con Egas Cueman ${ }^{1}$, pasando por la intervención más o menos puntual de Simón de Colonia $^{2}$ y la de los hermanos Egas $^{3}$ con quienes, según se desprende de las fuentes, se dio por concluida la fábrica en 1504, incluida la sacristía, actual lugar de acceso al convento.

1 Para una información más completa véase: J.M. AZCÁRATE RISTORI, "La fachada del Infantado y el estilo de Juan Guas", Archivo Español de Arte, 96 (1951), pp. 307-319; "El Maestro Hanequin de Bruselas", Archivo Español de Arte, 83 (1948), pp. 173-188; "La obra Toledana de Juan Guas", Archivo Español de Arte, 113 (1956), pp. 9-42; y la que fue su tesis doctoral, La arquitectura gótica toledana del siglo XV, Madrid, 1958; F. ARRIBAS ARRANZ, "Noticias sobre San Juan de los Reyes", Boletín del Seminario de Estudios de Arte y Arqueología, XXIX (1963), pp. 43-74. Algo más recientes son los trabajos de la profesora Pérez Higuera, véase: M.T. PÉREZ HIGUERA, "En torno al proceso constructivo de San Juan de los Reyes en Toledo", Anales de Historia del Arte, 7 (1997), pp. 11-24; y "San Juan de los Reyes", Castilla la Mancha-2. Toledo, Guadalajara y Madrid vol. 13 de la colección La España Gótica (Dir. Joan Sureda Pons), Madrid, 1998, pp. 114-130, muy semejante al anterior pero con nuevas aportaciones.

2 Entre otros trabajos, véase: F. ARRIBAS ARRANZ, "Simón de Colonia en Valladolid", Boletín del Seminario de estudios de arte y arqueología, II (1934), pp. 153-166. En este sentido y por su estrecha relación puede consultarse también: F.J. SÁNCHEZ CANTÓN, "El dibujo de Juan Guas. Arquitecto español del siglo XV”, Arquitectura, 115 (1928), pp. 339-347. Sobre este dibujo pueden consultarse, además, los siguientes artículos: S.L. SANABRIA, "A late gothic drawing of San Juan de los Reyes in Toledo at the Prado Museum in Madrid", Journal of the Society of Architectural Historians, 2 (1992), pp. 161-173; y E. HAMON, "Un dessin de la fin du Moyen Âge pour San Juan de los Reyes à Tolède", Bulletin Monumental, 2 (1993), pp. 420-422.

3 Esencial el artículo citado de Filemón Arribas de 1963. 
Con Enrique y Antón Egas entramos ya en el siglo $\mathrm{XVI}^{4}$ y con ellos se cerraría la etapa gótica del convento aunque no la de su construcción. El fallecimiento de la reina, ocurrido aquel mismo año, no supuso la paralización de las obras, continuándose el patronazgo regio por don Fernando e incluso por sus herederos, dado que en tiempos del Emperador tuvo un nuevo y fortísimo impulso. En esta época debió de comenzarse la construcción del segundo claustro del convento, concluido en tiempos de Carlos I, al que desde siempre se le ha atribuido ${ }^{5}$. Consta que en 1517 dicho claustro estaba, si no terminado, sí muy avanzado en su construcción, según podemos leer en la documentación existente y por la cual sabemos también que seguían estando en relación directa con la fábrica de San Juan de los Reyes, Enrique y Antón Egas, por tanto, podemos explicarnos el por qué de la continuación estilística ya bien entrado el siglo XVI.

En 1526, Carlos I expidió una cédula real por la que se libraba un pago para:

"acabar el retablo y rreja de la capilla prinçipal de la yglesia de dicho monasterio y los organos y portada d'ella"6.

Finalmente en mayo de 1534 se pagaba por hacer los "retablos laterales y las filateras de la dicha capilla y para las obras del dicho monasterios que están por acabar" y sobre todo, y lo más importante, por "acabar de hazer la portada de la dicha yglesia" ", aunque ésta se haría finalmente mucho más tarde.

Siguiendo con este breve repaso por los diferentes arquitectos que trabajaron en San Juan de los Reyes, en el siglo XVI también se construyó la caja de la escalera que comunica el claustro bajo con el alto ${ }^{8}$, muy posiblemente atribuible a los Egas y por tanto con una cubierta muy diferente, quizá igual o similar a la de la sacristía, es decir, una bóveda rebajada de crucería y combados, a la actual de casetones con florones. Ésta se atribuye tradicionalmente a Alonso de Covarrubias hacia el 1553, año en que el príncipe Felipe, daba un nuevo impulso a la portada de la iglesia que no se terminaría hasta comienzos del siglo XVII. Precisamente aquí es donde mejor se aprecia esta voluntaria continuidad estilística, al menos hasta llegar a los siglos XIX y XX.

4 Siguen estando aún vigentes las aportaciones sobre el periodo renacentista de F. MARÍAS, La arquitectura del Renacimiento en Toledo (1541-1631), t. III, Madrid, 1986.

5 P. DE SALAZAR, Cronica y Historia de la Fvundacion y progresso de la Provincia de Castilla, de la Orden del bienaventurado padre San Francisco, Libro III, Cap. II, Madrid, Imprenta Real, p. 141, dice: "Y algunas cosas que no quedaron hechas quando los Reyes murierõ, el Emperador Carlos Quinto de buena memoria su nieto las hizo, como son el retablo del altar mayor, que es suntuossisimo, y la reja de la Capilla mayor de hierro colorado dorada, poniendo las armas de los Reyes Catolicos sus abuelos a la mano derecha, y las de su Imperio a la yzquierda. Hizo el Claustro segundo, y otras muchas cosas".

6 F. ARRIBAS ARRANZ, op. cit., 1963, p. 71.

7 F. ARRIBAS ARRANZ, op. cit., 1963, p. 72.

$8 \mathrm{El}$ aspecto actual que ofrecen, tanto los peldaños como el pasamanos es debido a una campaña de restauración efectuada en 1954 a cargo de la Dirección General de Regiones Devastadas. 
La fachada actual, situada en el lado norte es el único acceso existente en el templo desde el exterior y ha sido bien estudiada y documentada por Fernando Marías. En opinión del autor, sería una obra de los canteros Francisco y Miguel del Valle según la traza de Juan Bautista Monegro que podría fecharse en torno a 1607 y 1609. Resulta muy interesante, por tanto, la disposición de la portada pues el lenguaje utilizado no corresponde con el estilo que cronológicamente le corresponde. Monegro parece querer mantener un goticismo impropio del momento que se aprecia muy bien en los arcos conopiales, así como en los laterales de la portada a modo de pilares baquetonados o sus remates en agudos pináculos -en cuyo caso ni tan siquiera pretenden ser semejantes a los de la obra de Guas. Un lenguaje mal entendido o no atrevido que desluce bastante en relación al resto y por cuyo motivo ha sido largo tiempo criticado en las fuentes. Pero no por ello deja de ser un interesante modo de mantener, a pesar del tiempo, el estilo original y propio de San Juan de los Reyes.

Más representativo aún si cabe de esta idea de continuación estilística sería el levantamiento de los dos campanarios o espadañas, el primero de ellos, sobre la fachada principal, contratado al maestro cantero Francisco del Vallen, según las indicaciones de Diego Alcántara, aparejador del Alcázar ${ }^{9}$. Según la descripción de Marías, el campanario:

"debía hacerse con tres vanos, entre cuatro pilares con pedestales, basas, capiteles y cornisas, claraboyas, remates y recuadros. Todos los ornamentos habrían de ser con molduras 'modernas' a la gótica, tanto los arcos como antepechos, y con la 'forma y proporción que al ydificio conviene', esto es, buscando su adaptación estilística y proporcional a la obra gótica de los Reyes Católicos"10.

El segundo campanario se levantó en 1592, en el lado oriental del templo, hacia el claustro principal y apoyado en el arranque del primer contrafuerte del presbiterio. Dio sus trazas Juan Bautista Monegro con el visto bueno de Juan de Herrera ${ }^{11}$. Según la descripción de Marías, este campanario:

“tenía los dos haces labrados, dos pilares con remate 'moderno' piramidal y relieves, fondos, resaltos, capiteles, basas, cornisamientos, remantes y fajas de labor moderna, bola y cruz".

El autor los menciona como derribados entre 1903 y 1904, pero el derribo se llevó a cabo años más tarde, pues en las fotografías de los años treinta se aprecia el gran arco de descarga situado a los pies de la torre que debía servir de apoyo al campanario, mientras que en las posteriores a los años cincuenta, dicho arco ya ha desaparecido.

Finalmente, siguiendo con este brevísimo repaso por los arquitectos que intervinieron en la construcción del convento de San Juan de los Reyes, señalo y avanzo que el claustro nunca llegó a terminarse del todo, al menos sus remates.

9 F. MARÍAS, op. cit., 1986, p. 90.

10 Ibidem.

11 Aporta toda la documentación relacionada con este campanario Ibidem, p. 90, notas 246 y 247. 
No se puede dejar de mencionar otro de los hitos importantes en la edilicia de San Juan de los Reyes: la construcción de la Capilla de la Beata Mariana de Jesús adosada al templo del convento en torno a $1727^{12}$. Capilla que se mantuvo en pie y supuso a los ojos de Ponz, Madrazo o Amador de los Rios, "un pegote feo y espantoso". Ponz dice exactamente de ella:

"junto a la puerta de esta iglesia de San Francisco hay una capilla labrada de sillares, y es de la Tercera Orden. Aunque no entré a verla, conocí su pésima fachada de escultura, y arquitectura lo que podrá ser por dentro"13.

No parece, pues, que en este momento interesara mantener el lenguaje de Juan Guas o tuvieran mayor respeto por la obra hecha que por la de ser un espacio sagrado. La capilla fue derribada en 1864 pero su portada, de gusto barroco, aún se conserva pues, una vez desmontada junto con sus esculturas, fue trasladada al Museo Provincial.

Ésta fue la última empresa constructiva llevada a cabo en San Juan de los Reyes. Sin embargo, un edificio de tales dimensiones y características debía de necesitar constantes reformas y reparaciones, como así venía sucediendo prácticamente desde su origen. Lamentablemente carecemos de noticias y datos de las obras que pudieron efectuarse durante la segunda mitad del siglo XVIII aunque es muy posible que, de haberlas realizado, éstas no fueran de gran envergadura sino más bien de mantenimiento y conservación.

El siglo XIX supuso un giro importante en el devenir de San Juan de los Reyes que vendría propiciado por el desarrollo de dos dramáticos acontecimientos políticos durante el primer tercio del siglo; por un lado la Guerra de Independencia y, por otro, la Desamortización. El primero, especialmente violento, el 19 de diciembre de 1808 cuando el mariscal Víctor ordenó no dejar ningún hospital en la ciudad y la evacuación de los heridos y enfermos a Madrid, momento en el que se produce el devastador incendio y destrucción del convento; y el segundo en 1836, que supuso el golpe de gracia al edificio. Las consecuencias de los mencionados acontecimientos históricos han sido tratadas con profundidad tanto por Antolín Abad Pérez ${ }^{14}$ como por Julio Porres Martín Cleto ${ }^{15}$, respectivamente, huelga, por tanto,

${ }^{12}$ Sobre la construcción de la capilla, véase: J. DE MORALEDA Y ESTEBAN, "La Beata Mariana. Su historia. Su capilla. Su supuesto retrato", Boletín de la Real Academia de Bellas Artes y Ciencias Históricas de Toledo, V (1923), pp. 190-195.

${ }_{13}$ A. PONZ, Viaje por España, en que se da noticia de las cosas más apreciables, y dignas de saberse, que hay en ella, tomo I, carta IV, Madrid, 1787, p. 166.

${ }^{14}$ A. ABAD PÉREZ, "Relación del incendio de San Juan de los Reyes (1808) y vicisitudes posteriores hasta 1864", Toletum, 75-80 (1969), pp. 169-188; "LA V.O.T. de San Juan de los Reyes y su capilla de la Beata Mariana", Anales Toledanos, 5 (1971), pp. 1-76; y "San Juan de los Reyes en su cincuenta aniversario de la restauración. 1954-2004", Antena Provincial, 123 (julio-octubre 2005), pp. 100-105.

15 J. PORRES MARTÍN CLETO, La Desamortización del siglo XIX en Toledo, Toledo, 1965; y "Los franciscanos en Toledo", Anales Toledanos, XVII (1983), pp. 17-28. Sobre este tema en particular puede consultarse, D. ORTIZ PRADAS, "La desamortización de San Juan de los Reyes y vicisitudes posteriores", 
extenderse en ambos temas pues es momento de entrar en materia, que versa, como se avanza en el título, sobre las diversas actuaciones que se produjeron en San Juan de los Reyes desde las de Arturo Mélida en el siglo XIX (1881) hasta los proyectos de Zabala, Carrasco y Muñoz, Moya o Valcárcel en el XX.

\section{Arturo Mélida, Maestro Mayor de San Juan de los Reyes}

La intervención de Arturo Mélida (1849-1902) en San Juan de los Reyes fue, sin lugar a dudas, la más importante e interesante de cuantas se hicieron a lo largo de los siglos XIX y XX en el convento. El proyecto del arquitecto madrileño, firmado y fechado en 1881, era del todo ambicioso y optimista en su parte teórica aunque poco realista en relación al presupuesto formado, dado el estado ruinoso del claustro y abandono del edificio. Sin embargo, es fácil suponer que Mélida ideara desde el principio una restauración completa, más allá de la simple reconstrucción material de las zonas dañadas, con la clara determinación de concluir con lenguaje similar la traza inacabada de Juan Guas. Es decir, actuó como lo habría hecho Viollet-le-Duc, a quien Mélida consideraba un maestro en el arte de la construcción, y para el que la restauración era "restablecer el edificio a un estado completo que puede nunca haber existido"16; pero también como lo exigía la legislación vigente en materia restauradora, dado que, como ya apuntara el profesor Navascués, la restauración monumental debía hacerse siempre respetando:

“el pensamiento primitivo, acomodando las renovaciones al carácter de la fábrica, y procurando que las partes antiguas y las modernas se asemejen y parezcan de la misma época""17.

Y así hizo Mélida en San Juan de los Reyes, ya que además de restaurar las zonas dañadas, restituyó fragmentos perdidos desde hacía años y, sobre todo, añadió, con el fin de dar al conjunto la coherencia que él creía indispensable, partes que nunca existieron. Con todo ello, Mélida dio al convento un claustro que jamás tuvo, un claustro ideal y utópico en el que se entremezclaban, en armonía con el conjunto, tanto la arquitectura como la escultura y la pintura, siendo pues, un perfecto ejemplo de obra de arte total y continuidad estilística.

Este sentimiento historicista se percibe desde su origen pues el proyecto mismo está inspirado en los documentos de la Edad Media, realizado en pergamino y

La Desamortización: el expolio del patrimonio artístico y cultural de la Iglesia en España, San Lorenzo de El Escorial, 2007, pp. 527-538, así como, "Los libros de viaje de los siglos XIX y XX como fuente para el estudio de la arquitectura medieval. San Juan de los Reyes de Toledo", J. MARTÍNEZ DE AGUIRRE y D. ORTIZ PRADAS (eds.), Cien años de investigación sobre arquitectura medieval española, Anales de Historia del Arte, vol. extraordinario (2009), pp. 347-362.

16 E. VIOLLET-LE-DUC, Dictionnaire raisonné de l'architecture française du XIe au XVIe siècle, París, 1854-1868 (10 vol.), concretamente, vol. VIII, 1866, p. 14.

17 Real Orden 10-X-1850, citado por P. NAVASCUÉS PALACIO, "Mélida y San Juan de los Reyes", Isabel la Católica. Reina de España, Madrid-Barcelona, 2002, pp. 331-355, p. 334. 
escrito imitando los caracteres medievales. Más allá del recurso efectista encontramos en la manera de actuar de Mélida una voluntad que trasciende lo puramente formal y que entronca directamente con el carácter de nuestro arquitecto, ya que el proyecto es, en palabras de Pedro Navascués:

"todo un reflejo de la actitud neomedieval de Mélida, del deseo de comprensión del monumento, del anhelo por conectar con la obra (...) del afán de incorporarse al taller de Juan Guas salvando el tiempo transcurrido"18.

Llegó a tal extremo la necesidad de identificarse con el propio arquitecto, de comprender sus razonamientos y hacerlos suyos, que no dudó en reclamar para sí, como así consta en el pliego de condiciones de la Memoria del proyecto, la condición de Maestro Mayor "con las mismas facultades de que estuvo revestido dicho cargo en la construcción del Monumento" 19 , buscando de este modo la misma consideración que Juan Guas en el siglo XV.

Son estos algunos ejemplos entresacados de la memoria que nos permiten conocer mejor la personalidad de Arturo Mélida y su manera de entender la restauración monumental y poder calificarlo como el arquitecto español más próximo y vinculado con la práctica restauradora francesa de Viollet-le-Duc.

A través de las fuentes gráficas ${ }^{20}$ conocemos el estado del claustro, elemento fundamental del convento, antes de la intervención de Mélida. El cotejo visual de éstas con la obra actual permite apreciar con claridad las aportaciones de Arturo Mélida, y a pesar de ello, han pasado en numerosas ocasiones desapercibidas. Dicho cotejo queda confirmado por la documentación conservada en diferentes archivos españoles. El proyecto de Mélida se podría dividir en tres fases: la primera, centrada en la restauración del claustro ${ }^{21}$; la segunda en la que se dotaría al convento de una nueva fachada y se intervendría en la sacristía y escalera principal del claustro y, finalmente, la actuación en la iglesia.

A la luz de la documentación existente podemos establecer los avances y logros obtenidos en la primera fase de restauración. La obra de Mélida se localiza fundamentalmente en el ámbito arquitectónico y se centró en la reparación del muro medianero con el segundo claustro, entonces solar de la futura Escuela de Artes, así como en la reconstrucción de la galería oeste y tres tramos de la norte,

18 P. NAVASCUÉS PALACIO, "Mélida y San Juan de los Reyes”, Isabel la Católica. Reina de España, Madrid-Barcelona, 2002, pp. 331-355, p. 333.

19 Ibidem.

20 Sobre el tema específico de la documentación gráfica de San Juan de los Reyes, puede consultarse: C.M. LARRA GONZÁLEZ, "San Juan de los Reyes y la fotografía histórica como método de análisis de su construcción e intervenciones", Fotografía y patrimonio: II encuentro en Castilla la Mancha, Ciudad RealToledo, 2007, pp. 198-205.

${ }^{21}$ La casi totalidad de la memoria quedó publicada en "Restauración del claustro de San Juan de los Reyes (arquitecto D. Arturo Mélida)", Boletín de la Real Academia de Bellas Artes de San Fernando, V (1885), pp. 226-233. 
y la reparación, sustitución y limpieza de casi la totalidad de los sillares y revestimientos del resto del claustro. En alzado la intervención de Mélida fue igual de amplia, haciendo de nuevo cuño y con diseño personal el remate y crestería del claustro, incluyendo una serie de gárgolas y pináculos, y sustituyendo la totalidad de los contrarrestos por otros de nueva labra reforzados. Por último, siguiendo los modelos existentes, muchos de ellos ya perdidos, Mélida desmontó e hizo nuevas tanto las tracerías de los veinte vanos del claustro bajo como los balaustres de los del claustro alto ${ }^{22}$.

Mayor interés presenta la solución aportada por el arquitecto en la cubierta del claustro alto (fig.1). En este sentido se debe insistir en que dicha cubierta es un diseño original de Mélida y no una restauración como algunos autores han apuntado. La reflexión del arquitecto en torno al modo de proceder en este caso, es decir, añadir al claustro un elemento completamente nuevo, es muy interesante especialmente porque supone un alarde de modernidad en materia de restauración monumental y por ser deudor por completo de los principios que sobre esta disciplina marcó Viollet-le-Duc. La decoración pictórica de la armadura es una muestra más del deseo del arquitecto de que la obra nueva fuera fiel reflejo

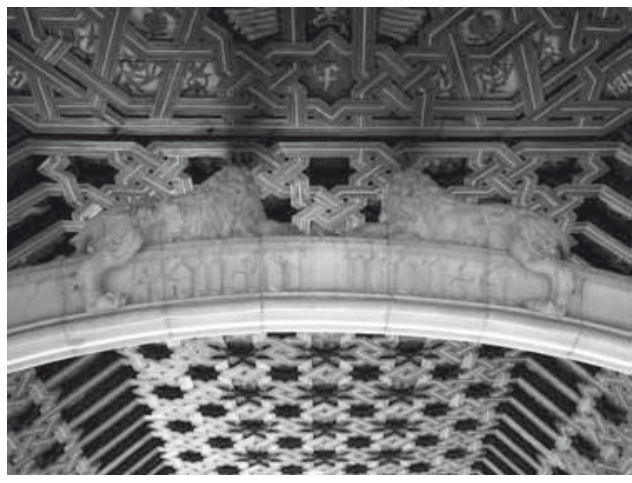

Fig. 1. San Juan de los Reyes, claustro, arcos de contrarresto del claustro alto, actualidad. de la antigua. Buscó recrear la aparente ingenuidad de la pintura medieval encargando la pintura, no a artesanos de renombre, sino a aprendices jóvenes para obtener el carácter naif de las obras antiguas y siempre enfatizando en los elementos decorativos para que no rompieran la coherencia y la unidad de estilo.

Se deben también a Mélida, como obra nueva y composición original, los arcos entibos situados en los extremos de cada crujía. Mélida buscó, como así lo recomendaba Viollet-le-Duc, en otras obras de Juan Guas soluciones similares que le sirvieran de ejemplo, las cuales encontró en la galería alta del patio del Palacio del Infantado de Guadalajara. No obstante, al no estar convencido del tipo y disposición de la decoración y sin querer copiar íntegramente el modelo, optó por introducir elementos originales aunque inspirados en la ornamentación hispanoflamenca.

22 El proyecto y memoria del presupuesto adicional puede consultarse en Archivo General de la Administración (=AGA), Sección de Educación (top. 31), Caja 8820, aunque la casi totalidad de la memoria quedó publicada también en S. ÁVALOS, "Presupuesto adicional del proyecto de Restauración del claustro de San Juan de los Reyes (1889)", Boletín de la Real Academia de Bellas Artes de San Fernando, V (1890), pp. 202209, si bien el texto impreso no incluye la documentación gráfica. 
Esta primera fase se alargó aproximadamente diez años, y concluidos en su gran mayoría todos los trabajos, Mélida ideó un segundo proyecto de renovación de la fachada del convento y restauración de la antigua sacristía y escalera principal ${ }^{23}$. Arquitectónicamente, y lo que más puede llamar la atención por entrar dentro del ámbito historicista, es la última parte del proyecto, es decir, la ejecución de una nueva fachada y el derribo del zaguán de entrada y traslado de la puerta principal, la llamada del Pelícano, de entrada al claustro. Estas actuaciones no pudieron ser llevadas a cabo por Mélida, pero sus planos sí fueron utilizados años más tarde, ya en el siglo XX, si bien sufrieron algunas modificaciones, pues el proyecto de fachada de Mélida era mucho más ornamental, acorde con el decorativismo del resto del edificio y siempre buscando la "unidad de estilo", que no llegó a concretarse por motivos presupuestarios.

Si la actuación de Mélida en el ámbito arquitectónico fue sustancial en la transformación de San Juan de los Reyes, no menos importante lo fue en el escultórico aunque bien es cierto que las aportaciones del arquitecto en este sentido se diluyen por las numerosas transformaciones que ha sufrido el edificio a lo largo de los años, especialmente en lo referente a las esculturas de bulto redondo que jalonan el claustro inferior, rehechas, restauradas o reubicadas en el siglo XX.

Distinta suerte corrió la recuperación de la iglesia del convento a lo largo del siglo XIX. Con la llegada de la Comisión Provincial de Monumentos en 1844, quedaron separados el templo, que quedaba en manos de la Iglesia, y el claustro, bajo tutela de la Comisión, existiendo entre ambos una división no sólo administrativa sino física, pues los accesos directos de la iglesia al claustro fueron condenados, es decir, el paso a las tribunas altas y la entrada correspondiente con la Puerta de la Verónica. En estos primeros años la recuperación de San Juan de los Reyes, tanto del claustro como de la iglesia corrían parejos, situación que variaría drásticamente a partir de 1881 a favor del claustro, donde se centraron todos los esfuerzos, quedando la iglesia al margen de esta situación ${ }^{24}$. Sin embargo, a lo largo de los últimos años del siglo XIX, se percibe la necesidad, cada vez más acuciante, de dar comienzo a la restauración definitiva del templo y de este modo poner fin al progresivo deterioro que lo amenazaba.

Según fuentes posteriores ${ }^{25}$, en 1898 se dio comienzo a un proyecto de restauración integral de la iglesia, sin dar más datos, que continuaría hasta bien entrado el

23 A. MELIDA, "Proyecto de reparaciones de la crujía que ocupa el Museo Provincial y la escalera de Covarrubias", Archivo de la Academia de BB.AA. de San Fernando, Leg. 192-5/5.

${ }^{24}$ Lamentablemente a la hora de tratar el tema de la restauración de la iglesia de San Juan de los Reyes, nos encontramos con un problema añadido, pues al ser propiedad de la Iglesia, las competencias en materia de restauración no eran del Ministerio de Fomento, sino de la Junta de Culto y Clero y su afín Junta de Reparación de Templos, con carácter provincial y bajo la dirección de un Arquitecto diocesano dependiente del Ministerio de Gracia y Justicia. Sobre este asunto puede consultarse, J. MARTÍN SÁNCHEZ, "La tutela de la arquitectura religiosa y la supresión del diezmo", La Desamortización: el expolio del patrimonio artístico y cultural de la Iglesia en España, Actas del XV simposium de Estudios Superiores de El Escorial, San Lorenzo de El Escorial, 2007, pp. 57-76.

25 En los proyectos de restauración de los años cincuenta a cargo de la Dirección General de Regiones Devastadas. 
siglo XX. Por la fecha indicada podría corresponder con una posible actuación de Mélida, pero hasta el momento no hemos podido documentarla.

Con esta hipotética intervención de Arturo Mélida nos adentraríamos de pleno en el siglo XX, donde los diferentes arquitectos se fueron sucediendo habiendo perdido gran parte de ese sentimiento historicista pero con la clara intención de seguir manteniendo una perceptible unidad de estilo para no dejar la obra de Juan Guas desvirtuada.

\section{La intervención de Manuel Zabala y Gallardo}

Al fallecer Mélida sin finalizar la restauración, quedó "la iglesia apuntalada, entibado su coro y andamiado su muro oeste" 26 (fig. 2). La labor restauradora

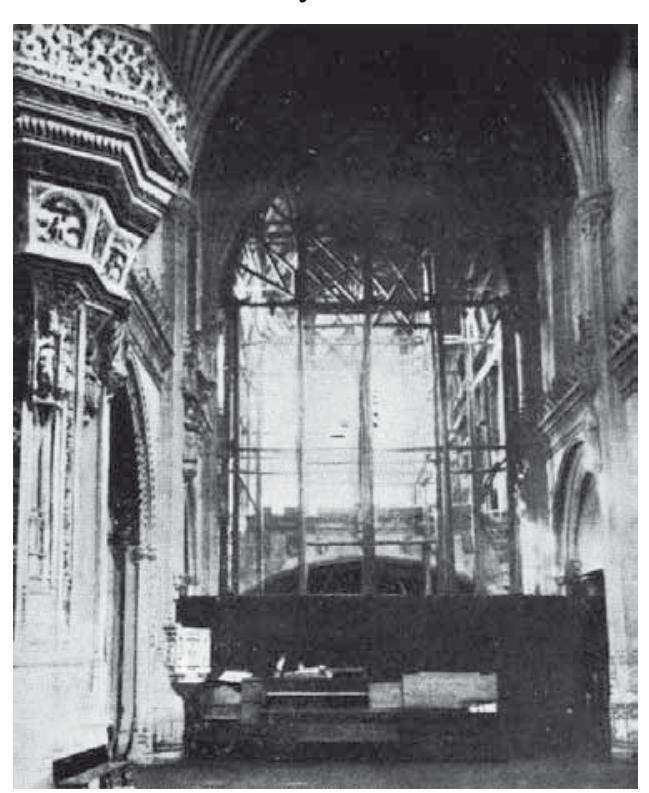

Fig. 2. San Juan de los Reyes, "Interior de la iglesia de San Juan de los Reyes, sotocoro apuntalado”, h. 1927 [tomado de la revista Toledo, $\mathrm{n}^{\circ} 243$ (1927), p. 1669] fue continuada por Manuel Zabala y Gallardo $^{27}$, de la Junta Facultativa de Construcciones Civiles quien, con graves problemas presupuestarios, logró reponer en una primera fase los pináculos de la fachada norte. Las fotografías del momento permiten ver cómo aquellos contrastaban violentamente con la oscura piedra de los contrafuertes que los sustentaban, todavía no restaurada.

En $1921^{28}$ el mismo arquitecto redactó un nuevo proyecto que amplió por tercera vez en $1928^{29}$. En esta fecha los progresos alcanzados en el exterior de la iglesia eran evidentes. Casi la totalidad de los pináculos de la iglesia, tanto los de sus fachadas norte y sur como los del cimborrio, estaban restaurados, habiéndose sustituido por otros

26 "De las obras de San Juan y Santa Cruz", Toledo, 59 (1916), 6.

27 No sabemos con exactitud el momento de su nombramiento, pero no debió de demorarse mucho tiempo. En junio de 1903 y por R.D. de 29 de mayo, se nombraba al arquitecto Manuel Zabala y Gallardo, junto a Ricardo Velázquez Bosco, Simeón Ávalos, Fernando Arbós y Federico Aparici, Vocal de la Junta facultativa de Construcciones Civiles y Secretario de la misma.

28 No conservado en la actualidad.

29 M. ZABALA GALLARDO, "Proyecto de obras para continuar las de conservación y reparación que se ejecutan en la iglesia de san Juan de los reyes", Archivo General de Administración (A.G.A.) (5)14 $31 / 4895$ y $31 / 4894$. 
de nueva labra de piedra granítica ${ }^{30}$ siguiendo modelos originales. La crestería del primer cuerpo de la fachada norte se había sustituido por completo por una nueva, así como parte del antepecho que recorría la cabecera, devolviéndole su aspecto de tracería calada.

Sin embargo, a pesar de los logros obtenidos por Zabala, a su muerte, ocurrida en 1934, aún quedaba mucho por hacer, especialmente en el interior del templo cuyo coro seguía apuntalado - pues el descabalgado de sus piezas hacía peligrar su seguridad- y, por lo tanto, tampoco se había colocado la nueva balaustrada de cerramiento. Las capillas anejas, al igual que el sotocoro, mantenían todavía el apuntalamiento de las bóvedas y el cimborrio, con profundas grietas y graves problemas de estabilidad, ni tan siquiera se había previsto su reparación en esta primera fase del proyecto. Y puesto que los problemas estructurales no estaban solucionados, era evidente que los decorativos estaban en un segundo plano.

\section{La intervención de Emilio Moya Lledós}

La salud de Zabala debía ser muy frágil, pues un año antes de su muerte, otro arquitecto, también Vocal de la Junta de Construcciones Civiles, se hizo cargo de las obras de restauración de San Juan de los Reyes. En efecto, en marzo de 1933, Emilio Moya LLedós (1894-1943) ${ }^{31}$ redactó un proyecto de "Obras de consolidación en la iglesia de San Juan de los reyes de Toledo" 32 cuya finalidad "era la terminación de las partes altas de la iglesia para poder quitar el andamio". El proyecto comprendía también y por primera vez la "consolidación de las tres grandes bóvedas del refectorio"33 (fig. 3 ), aunque esta última parte no se llevaría a efecto hasta los años cincuenta. No

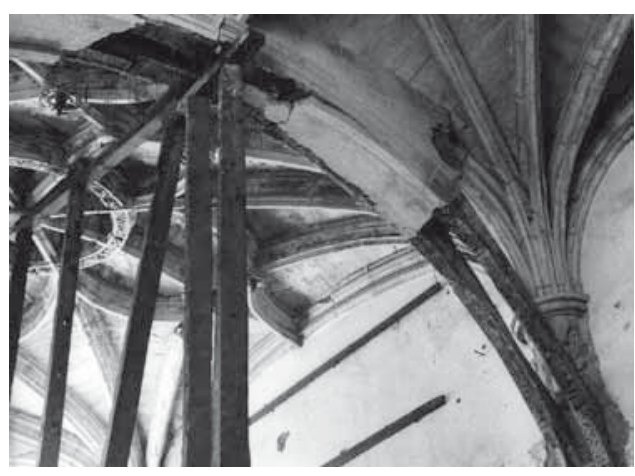

Fig. 3. San Juan de los Reyes, interior, detalle del sotocoro apuntalado, 1954, A.G.A.

30 Sin duda, la elección de los materiales estuvo errada. La variedad de canteras utilizadas a lo largo del tiempo - la de la Rosa agotada desde hacía poco tiempo- y la poca idoneidad de la piedra utilizada por su diferente tonalidad y comportamiento ante los agentes atmosféricos hace que todavía hoy sean excesivamente visibles las diferencias, dando como resultado un violento contraste entre la piedra original y la restaurada.

31 Emilio Moya desarrolló una ingente labor durante el período que tuvo la responsabilidad de la conservación de monumentos en la 4. ' Zona, de la que dan buena muestra las 117 intervenciones llevadas a cabo por él entre principios de 1932 y el primer trimestre de 1936. Véase J. ESTEBAN CHAPAPRÍA, "Emilio Moya LLedós, arquitecto conservador de monumentos (1929-1936)", Roma y la tradición de lo nuevo. Diez artistas en el Gianicolo (1923-1927), Madrid, 2004, pp. 118-129, p. 123.

32 Aprobado por R.D de 8 de junio de 1933, publicado en la Gaceta el 18 de junio de ese mismo año.

33 E. MOYA, "Proyecto de Obras de consolidación en la iglesia de San Juan de los reyes de Toledo", A.G.A., sig. (5) 14 31/4893. 
obstante, Moya vio truncada su labor en San Juan de los Reyes y su carrera, a partir de la Guerra Civil.

\section{San Juan de los Reyes y la Dirección General de Regiones Devastadas (1938-1957)}

La Guerra Civil española (1936-1939) supuso un paréntesis en el proceso de restauración de San Juan de los Reyes. Los años de la contienda fueron un duro golpe para el patrimonio español; ciudades y pueblos fueron arrasados por los bombardeos indiscriminadamente. Toledo sufrió de forma considerable, especialmente durante el asedio al Alcázar, que duró algo más de dos meses, y significó la destrucción total del edificio y de los alrededores ${ }^{34}$.

Aún no concluida la contienda, el bando nacionalista creó en marzo de 1938, con evidentes fines propagandísticos, el Servicio Nacional de Regiones Devastadas y Reparaciones con el objeto de ocuparse de la dirección y vigilancia de todas aquellas reconstrucciones que se llevaran a cabo por daños ocasionados por la guerra. Al año siguiente pasó a depender del Ministerio de la Gobernación convirtiéndose en Dirección General. De este modo, cualquier expediente de reconstrucción, ya fuera de bienes particulares o públicos, debía ser tramitado a través de ella, convirtiéndose en un instrumento más del Régimen. Tras una importantísima labor que duró casi dos décadas, la Dirección General de Regiones Devastadas desapareció en 1957 cuando la reconstrucción se dio por finalizada ${ }^{35}$, aunque en la práctica seguía funcionando.

La Dirección General de Regiones Devastadas editó la revista Reconstrucción donde quedaron recogidos, en muchos de sus artículos, los criterios de restauración que se debían seguir, difundiendo al mismo tiempo la labor emprendida por ella entre los años 1940 y 1956. Toledo fue, junto con otras ciudades como Madrid o Belchite, centro de atención preferente de la Dirección General. Se tuvo que reconstruir el Alcázar, prácticamente desde sus cimientos, así como la Plaza de Zocodover, destruidos la mayor parte de sus edificios por los bombardeos. Todas estas empresas de restauración quedaron reflejadas en la revista, donde se proponen actuaciones encaminadas a recuperar el aspecto más pintoresco de la ciudad, optando entonces por una restauración que podríamos denominar en "estilo", reinventando,

34 Véase sobre el tema, entre otras monografías: R. BALLESTER ESCALAS, El Alcázar de Toledo (La Guerra Civil Española, vol.2), Madrid, 1972; J. ÁLVAREZ LOPERA, La política de Bienes Culturales del gobierno republicano durante la Guerra Civil Española, Madrid, 1982; Á. PALOMINO, Defensa del Alcázar: una epopeya de nuestro tiempo, Barcelona, 1998; y J.M. RUIZ ALONSO, La guerra civil en la provincia de Toledo, Ciudad Real, 2004.

35 E. LLANOS DE LA PLAZA, "Dirección General de Regiones Devastadas", Arquitectura en Regiones Devastadas, Madrid, 1987, pp. 35-44. Véase también D. PERIS SANCHEZ, La Modificación de la ciudad. Restauración monumental en Toledo siglos XIX y XX, Toledo, 2009, pp. 81-125. 
de algún modo, el Alcázar, el Arco de la Sangre y otros muchos edificios más en una suerte, y con todas las reservas, de "neo-romanticismo".

En relación a San Juan de los Reyes, la fortuna y su posición al otro extremo de la ciudad con respecto al Alcázar, evitaron que fuera dañado durante los bombardeos, sin sufrir otro percance -al menos documentado- que la pérdida de gran parte de las tejas de las cubiertas y, sobre todo, la paralización de las obras el tiempo que duró la guerra, pero pronto entró a formar parte del grueso de monumentos cuya restauración se hacía necesaria.

\section{José Manuel González Valcárcel Arquitecto Conservador de San Juan de los Reyes}

En este sentido, y siguiendo con la lista de arquitectos de San Juan de los Reyes, el primer proyecto de restauración y rehabilitación del convento tras la Guerra Civil estaría a cargo del arquitecto José Manuel González Valcárcel (1913-1992), dependiente del Ministerio de Educación y no de la Dirección General. En noviembre de 1940 y a los pocos días de haber logrado su título fue nombrado Arquitecto Conservador de Toledo y como tal fue el encargado de llevar a cabo la restauración y conservación de numerosos edificios de la provincia y su capital. Fue González Valcárcel quien, en mayor o menor medida, y no siempre respetando el valor artístico de los monumentos, restauró el Taller del Moro, la Casa-Museo de El Greco, la Catedral, el monasterio de Concepción Franciscana y las iglesias de Santiago del Arrabal, Santa Eulalia, San Román, San Lucas, Santa Leocadia, Santa Isabel de los Reyes y, por supuesto, la de San Juan de los Reyes.

Las primeras obras efectuadas por Valcárcel ${ }^{36}$ fueron de bajo presupuesto y estuvieron encaminadas exclusivamente a la conservación, sin añadidos de ningún tipo; en proyectos posteriores de mayor envergadura sí los habría, comenzando con la reconstrucción de la parte conventual de San Juan de los Reyes. En efecto, en abril de 1941, el Ministerio de Educación Nacional, autorizaba la instalación de una comunidad franciscana en San Juan de los Reyes, aunque precisando que en usufructo -como sigue siéndolo en la actualidad. La Comunidad franciscana acordó solicitar de la Dirección General de Bellas Artes la construcción de un pabellón de nueva planta que sirviera de aula a los estudiantes de Teología. Al no poder asumir esta Dirección la reconstrucción de la iglesia y levantar un nuevo pabellón, se dirigió a la Dirección General de Regiones Devastadas, que aceptó sin vacilar el nuevo proyecto. A partir de entonces, la reedificación del convento correría a cargo de los arquitectos Francisco de Echenique y José Losada Barroso, adjuntos

36 J. GONZÁLEZ VALCÁRCEL, "Proyecto de obras de conservación en el convento de San Juan de los Reyes. Cubiertas”, mayo de 1941, A.G.A., sig. (3)115 26/00382. 
a la Dirección General, y la iglesia, restaurándose en paralelo, estaría en manos de José González Valcárcel.

Así pues, en 1949, se daba inicio al proyecto de reconstrucción del convento de San Juan de los Reyes con sus nuevas dependencias habitacionales y docentes y el encargado de llevarlo a cabo sería Francisco Echenique Gómez ${ }^{37}$ (fig. 4). Lo más destacado del proyecto, en relación con el edificio histórico que nos ocupa, es la reaparición del propósito de derribar la antigua portería del convento y trasladar la Puerta del Pelícano (fig. 5) a un nuevo lugar y, ya en la iglesia, reabrir la puerta de entrada en la fachada oeste que desde hacía tiempo estaba tapiada. Se preveía también la consolidación de las bóvedas de la planta baja de la Sacristía y la sustitución del solado de la primera. El proyecto seguía, en definitiva, lo propuesto por Mélida medio siglo antes.

En 1950, Valcárcel firmó otro proyecto de restauración de la iglesia del convento $^{38}$. Según se desprende de las diferentes memorias de intervención, la obra, que

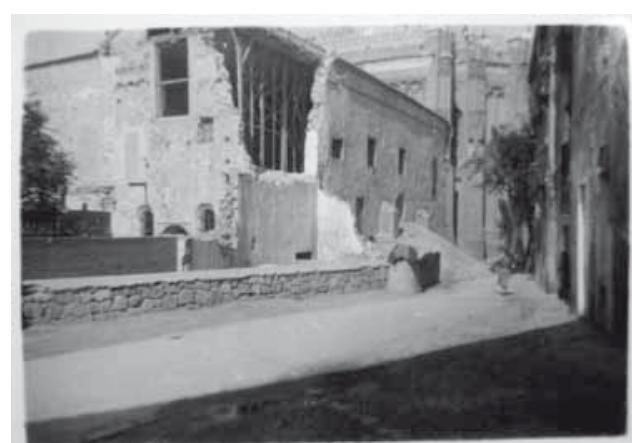

Fig. 5. San Juan de los Reyes, exterior, derribo de la Puerta del Pelícano, 1954 (Foto de José Aguado Villalba, cedida por Rosalina Aguado).

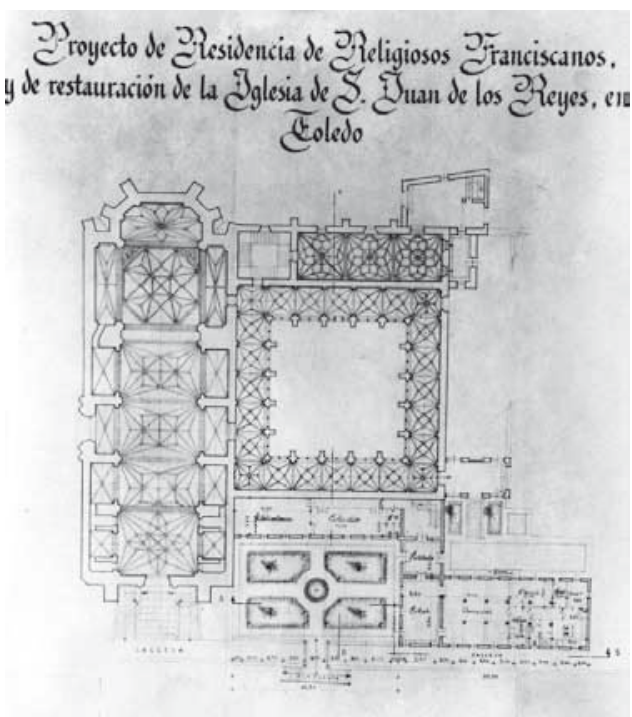

Fig. 4. Francisco Echenique, Proyecto de reconstrucción de San Juan de los Reyes, planta del convento, 1953. A.G.A. tenía que haber consistido en una simple sustitución de los pares y tirantes de la cubierta, se convirtió en algo más aparatoso que todo ello, pues todo el entramado del trasdós de las bóvedas se sustituyó por vigas de hierro. Se desmontaron las bóvedas y se engatillaron sus nervios con pletinas de hierro, algunas de las cuales ahora están a la vista. En el coro, el arco frontal fue desmantelado en su totalidad, así como parte de la plementería, nervios, ligaduras, terceletes y contraligaduras de las bóvedas del coro bajo

37 F. ECHENIQUE GÓMEZ, "Proyecto de reconstrucción del convento de Padres Franciscanos en el monasterio de San Juan de los Reyes”, A.G.A., sig. (4) 111, CA. 1590.

38 J.M. GONZÁLEZ VALCÁRCEL, "Proyecto de restauración en la iglesia de San Juan de los Reyes de Toledo", A.G.A., sig. (5) 14 31/6008. 
al haber sido reparadas muchas de sus dovelas con yeso y agramiladas. En cuanto a la parte artística, se completaban las obras eliminando el tabique que cegaba el ventanal del hastial oeste y colocando unas vidrieras de simetría llevando como elemento decorativo los anagramas de Isabel y Fernando. Es decir, como podemos verlo en la actualidad.

\section{La reconstrucción del claustro por Francisco Echenique y José Losada}

Parece, aunque no hay documentación que así lo confirme, que a partir de 1953 la Dirección General de Regiones Devastadas se hizo cargo de la totalidad de las obras ejecutadas en San Juan de los Reyes.

En esta fecha el arquitecto Echenique redactó la segunda fase del "proyecto de reconstrucción de San Juan de los Reyes", dada la necesidad de ampliar las obras y el presupuesto fijado en $1949^{39}$. El proyecto resulta de extrema importancia por cuanto supone un cambio sustancial en la fisionomía del edificio, no sólo su parte arquitectónica, sino también la decorativa. Debemos reconocer el mérito que merece el proyecto, pues las obras previstas que se continuaron en un sentido o en otro más de diez años, dieron a San Juan de los Reyes el aspecto que hoy ofrece, completando por fin la restauración que Arturo Mélida inició.

Más interesante para nuestro propósito resulta el capítulo añadido en esta segunda fase del proyecto sobre la necesidad de reparar el claustro. El presupuesto supone una información de primer orden, pues se dice que al menos se prevé hacer "12 imágenes completas de 1,25m altura talladas en piedra jarreta" y restaurar por completo otras 25 , lo que supone un total de 37 esculturas, de un total de 56, que o son nuevas o han sido modificadas, es decir, casi el $66 \%$ de las estatuas del claustro bajo. La talla y restauración de las esculturas se llevarían a cabo a partir de 1959, cuando por necesidades presupuestarias se elabore un tercer proyecto.

En el exterior del edificio también se realizaron importantes trabajos como queda reflejado en la memoria y presupuestos. Se sustituyeron por completo los antepechos calados del primer cuerpo de la cabecera, y se repararon los paramentos con la colocación de nuevos sillares. Se colocaron nuevos los pináculos del ochavo del cimborrio y, lo que es más significativo, se sustituyeron algunos de los añadidos por Mélida, por encontrarse en muy mal estado o ya desmochados.

Al igual que en el claustro, el exterior del templo contaba con magníficas esculturas de cuerpo entero y tamaño natural representando heraldos de los Reyes Católicos con sus armas y reinos. Vemos cómo estaba prevista la ejecución de tres

39 F. ECHENIQUE, "Proyecto de reconstrucción de San Juan de los Reyes (2 ${ }^{\mathrm{a}}$ Fase)", A.G.A., sig. (4) 111, CA 20647. TOP 76/13. 
de ellos y la restauración de otros nueve. Finalmente se preveía la restauración de las esculturas de la portada norte de la iglesia ${ }^{40}$.

Todas las obras de cantería y talla artística estarían a cargo de los Talleres Béjar de Toledo por contratación directa. Estaban constituidos por tres hermanos: Tomás, Federico y Cecilio, éste último como artista probado, profesor de la Escuela de Artes y Oficios, llevaba la dirección de los trabajos, y creó un equipo de canteros-artistas que fueron labrando cada una de las piezas necesarias.

\section{San Juan de los Reyes en la actualidad}

De este modo se daba por concluida la restauración de San Juan de los Reyes que duró casi un siglo rescatándolo de la ruina y una segura destrucción. Los trabajos que desde los años ochenta se han realizado en el convento toledano han sido más de conservación que de restauración y no han añadido elementos que puedan causar confusión o controversia. Para terminar, citaremos la obra de Carlos Baztan Lacasa, la memoria y estudio estructural del cimborrio del profesor Santiago Huerta y el último proyecto realizado por el arquitecto Javier Contreras, que ha finalizado hace poco tiempo.

40 Ibidem. 